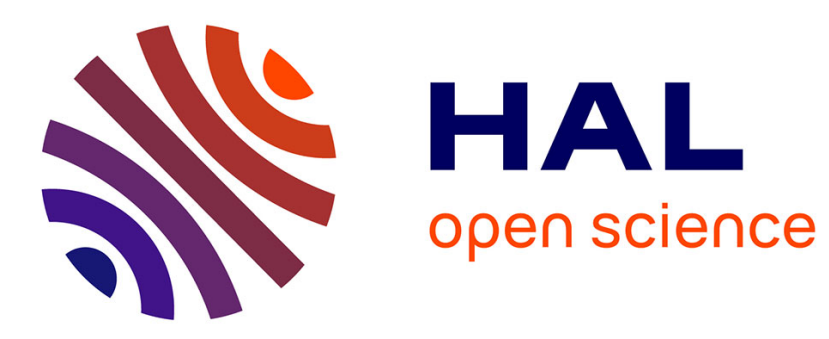

\title{
Simulation cinétique du phénomène d'agrégation
}

C. Allain, B. Jouhier

\section{To cite this version:}

C. Allain, B. Jouhier. Simulation cinétique du phénomène d'agrégation. Journal de Physique Lettres, 1983, 44 (11), pp.421-428. 10.1051/jphyslet:019830044011042100 . jpa-00232212

\section{HAL Id: jpa-00232212 https://hal.science/jpa-00232212}

Submitted on 1 Jan 1983

HAL is a multi-disciplinary open access archive for the deposit and dissemination of scientific research documents, whether they are published or not. The documents may come from teaching and research institutions in France or abroad, or from public or private research centers.
L'archive ouverte pluridisciplinaire HAL, est destinée au dépôt et à la diffusion de documents scientifiques de niveau recherche, publiés ou non, émanant des établissements d'enseignement et de recherche français ou étrangers, des laboratoires publics ou privés. 
Classification

Physics Abstracts

05.40

\title{
Simulation cinétique du phénomène d'agrégation
}

\author{
C. Allain et B. Jouhier \\ Laboratoire d'Hydrodynamique et de Mécanique Physique (*), \\ E.S.P.C.I., 10, rue Vauquelin, 75231 Paris Cedex 05, France
}

(Reçu le 30 novembre 1982, accepté le 12 avril 1983)

\begin{abstract}
Résumé. - La mise en place d'une expérience de simulation à deux dimensions nous a permis d'étudier la formation d'amas au cours du phénomène d'agrégation. L'existence d'une fraction surfacique critique et de propriétés géométriques d'homothétie interne des amas a été mise en évidence.
\end{abstract}

\begin{abstract}
An experimental simulation in two dimensions has permitted us to study the growth of clusters during an aggregation process. The existence of a critical surface fraction and of selfsimilar geometrical properties of the clusters is demonstrated.
\end{abstract}

Les processus d'agrégation, c'est-à-dire la formation d'amas à partir d'unités élémentaires initialement isolées, interviennent dans des domaines aussi variés que le génie chimique (flocculation des colloïdes, déstabilisation des émulsions, gels chimiques), la biologie (ségrégation des protéines dans les membranes) ou même la météorologie (formation des nuages). La difficulté de modélisation de ce processus provient de ce qu'il est intrinsèquement cinétique et donc dans une situation hors d'équilibre. En particulier les théories thermodynamiques des transitions de phase ou statistiques de percolation ne seront pas bien adaptées à ce type de problème.

Une autre approche consiste à écrire l'ensemble des équations d'évolution du système. La formation irréversible, à partir d'amas de taille $m$ et $n$, d'un amas de taille $p=m+n$, s'écrit schématiquement :

$$
m+n \rightarrow p
$$

Soit $N_{m}$ le nombre d'amas de taille $m$. L'évolution de $N_{m}$ peut s'écrire sous la forme d'une équation de Smoluchowski [1-3] :

$$
\frac{\mathrm{d}}{\mathrm{d} t} N_{m}=\frac{1}{2} \sum_{i+j=m} K_{i j} N_{i} N_{j}-\sum_{i=1}^{\infty} K_{i m} N_{i} N_{m},
$$

où $K_{i j}$ est appelé matrice des coefficients cinétiques.

Dans la suite nous supposerons que la réaction (1) se fait sans énergie d'activation; les coefficients $K_{i j}$ sont alors complètement déterminés par la probabilité de collision entre un amas de taille $i$ et un de taille $j$. Suivant que le système est contrôlé par la diffusion brownienne ou les

(*) E.R.A. 1000 . 
mouvements hydrodynamiques, les coefficients cinétiques $K_{i j}$ n'ont pas la même valeur et le processus d'agrégation est qualifié respectivement de péricinétique (P) et d'orthocinétique (O) [4]. La limite entre ces deux cas peut être définie à partir d'un critère adimensionnel :

$$
A=\frac{\tau_{\mathrm{b}}}{\tau_{\mathrm{c}}} \quad A<1(\mathrm{P}) ; A>1(\mathrm{O}),
$$

où $\tau_{b}$ et $\tau_{c}$ sont les temps caractéristiques entre collisions dans les cas respectivement d'une diffusion brownienne et d'un cisaillement.

Pour un système dilué le critère $A$ s'écrit [5] :

$$
A=\frac{\left(R_{i}+R_{j}\right)^{2}}{D_{i}+D_{j}} G
$$

où $R_{i}$ est le rayon d'un amas de taille $i$ et $D_{i}$ son coefficient de diffusion; $G$ représente le gradient de cisaillement. Pour chaque taille de particules on peut donc définir une valeur du cisaillement au-delà duquel les effets hydrodynamiques prédomineront. Par exemple, dans le cas de l'eau, pour $R_{1}=1 \mu$ le gradient critique sera $1 \mathrm{~s}^{-1}$ et pour $R_{1}=0,2 \mu$ il sera $100 \mathrm{~s}^{-1}$. Soulignons qu'un flocculateur classique ou un agitateur magnétique induisent des cisaillements de l'ordre de $100 \mathrm{~s}^{-1}$, c'est-à-dire que le milieu se situera le plus souvent dans la zone intermédiaire entre une agrégation péricinétique et orthocinétique.

Dans le cas où le système est concentré la situation est beaucoup plus complexe aussi bien pour le cas où le système est au repos (les processus de diffusion brownienne dans les systèmes concentrés étant difficiles à décrire) que pour le cas où il est soumis à un cisaillement. Il n'est pas évident par ailleurs que l'équation (2) qui néglige les corrélations spatiales soit encore valable.

Afin d'étudier cette situation nous avons construit une expérience de simulation macroscopique à deux dimensions. Les unités élémentaires sont des billes de cire de diamètre $1,8 \mathrm{~mm}$ en flottation sur l'eau. A cause de la tension interfaciale, lorsque deux billes entrent en collision elles restent en contact. La force d'attraction qui est simple à calculer dans le cas de deux plans [6] est plus subtile dans le cas de deux sphères. Cependant il est possible de démontrer qu'elle est encore attractive. Au début de l'expérience les billes sont réparties aléatoirement sur la surface. Pour cela nous engendrons à l'intérieur de l'eau des bulles de faible dimension qui, arrivant à la surface, cassent les amas préexistants. Après arrêt de l'agitation la formation des amas est observée sur plusieurs heures. Par exemple la photo 1 a été prise $1700 \mathrm{~s}$ après l'arrêt de la formation des bulles. L'analyse de clichés pris à différents temps et pour différentes fractions surfaciques de billes nous a permis d'obtenir les résultats suivants :

\section{Existence d'une fraction surfacique critique.}

Lorsque la fraction surfacique $\phi$ des billes est inférieure à une certaine valeur critique $\phi_{c}$, nous n'observons pas d'amas s'étendant d'un bord à l'autre du récipient alors que, lorsque $\phi$ est plus grand que $\phi_{c}$, il apparaît au bout d'un temps fini un amas de la dimension du récipient. Ce comportement est analogue à ce qui est observé en percolation [7] où, pour une probabilité d'occupation des sites supérieure à une probabilité critique, il apparaît un amas infini. La surface que nous utilisons étant rectangulaire $(40 \mathrm{~cm}$ par $65 \mathrm{~cm})$, nous avons choisi comme critère l'existence d'un amas s'étendant d'un bord à l'autre de la cuve suivant la petite dimension. Nous avons observé une fraction surfacique critique de $\phi_{c}=0,125 \pm 0,03$ ce qui correspond à environ 12500 billes L'importance de la barre d'erreur est due au fait que les billes ont tendance à s'agglomérer le long des parois du récipient sur quatre ou cinq rangs ce qui modifie le nombre de billes disponibles.

Cependant, soulignons que, à l'opposé du cas de percolation, il n'est pas évident que la valeur critique de $\phi$ ne dépende pas des dimensions relatives du bac et des billes. Dans la suite de cette étude nous n'avons étudié que le cas d'une concentration en surface égale à $\phi_{\mathrm{c}}$. 


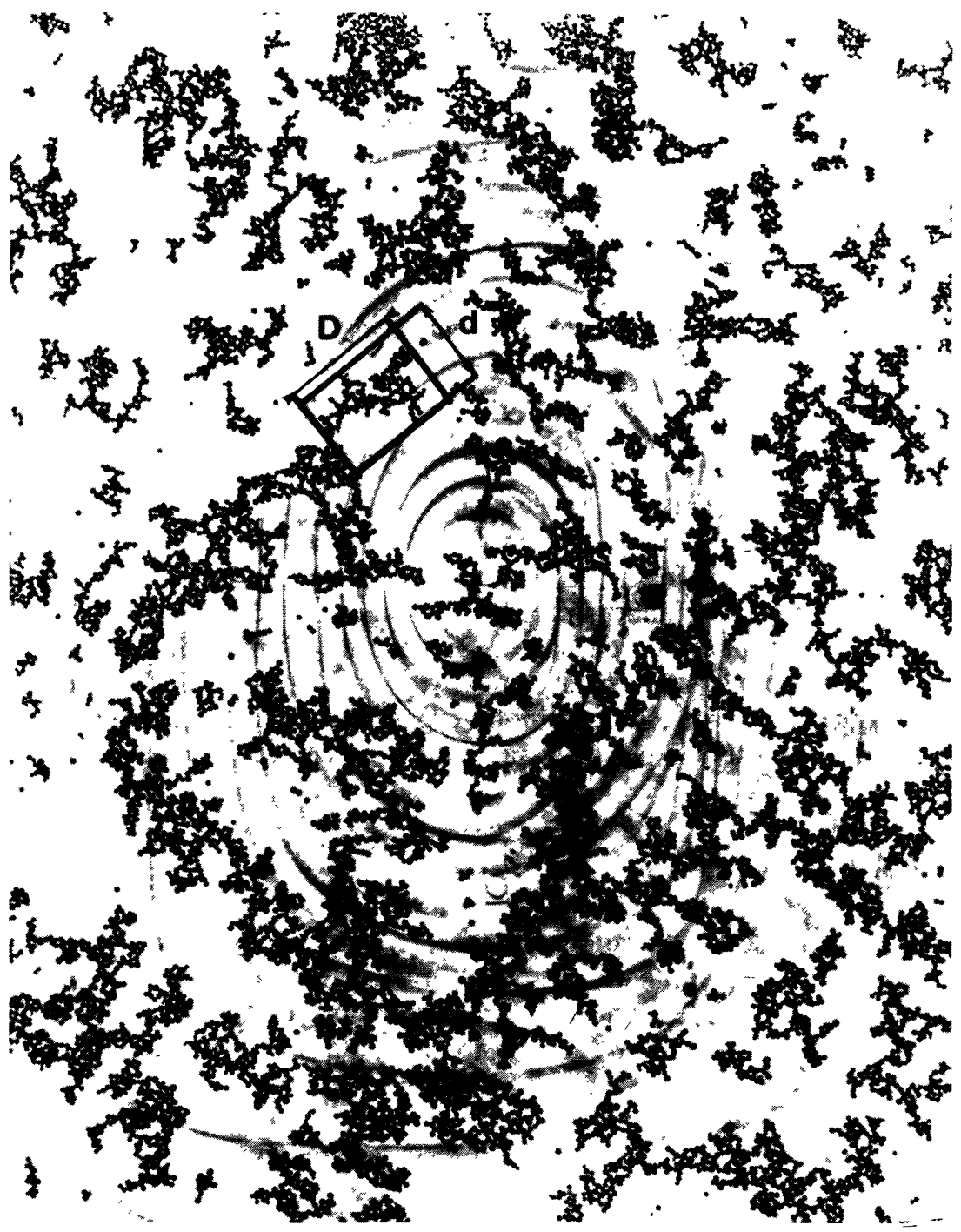

Fig. 1. - Cliché pris $1700 \mathrm{~s}$ après le début de l'expérience. On observe des amas de billes (particules noires) de dimension importante. Le serpentin qui apparaît au fond de la cuve est simplement un tuyau percé de nombreux trous très fins par lesquels s'échappent, lorsqu'on injecte de l'air dans le tuyau, les bulles servant à casser les amas. Le début de l'expérience correspond à l'arrêt de l'arrivée de l'air.

[Picture taken $1700 \mathrm{~s}$ after the beginning of the experiment. Large sized clusters of balls (dark particles) are observed. The tube at the bottom of the basin is a pipe drilled with a large number of very thin holes. When air is injected into the pipe, bubbles are formed and break the preexisting clusters when they reach the surface. The beginning of the experiment corresponds to the end of the injection of air.] 


\section{Nature du mouvement.}

En analysant des clichés pris à des intervalles de temps suffisamment proches (100 s) on peut déterminer les trajectoires des différents amas. Nous avons observé que, alors que certaines zones de la surface sont quasiment au repos, d'autres présentent des mouvements d'ensemble dus vraisemblablement à la présence de mouvements hydrodynamiques résiduels dans la cuve. L'analyse de la série de clichés, dont sont extraites les photos de la planche 2, montre des cisaillements compris entre 0 et $10^{-2} \mathrm{~s}^{-1}$ avec une valeur moyenne d'environ $10^{-3} \mathrm{~s}^{-1}$. Afin de déterminer si les effets de cisaillement sont prépondérants ou non dans le processus d'agrégation que nous étudions, nous pouvons utiliser le critère présenté au début de cette note (Eq. (4)). Cependant, il n'est pas trivial dans notre cas de déterminer des coefficients de diffusion. Dans les zones de très faibles cisaillements on observe bien des trajectoires en ligne brisée mais la concentration en surface étant très élevée, un amas sera entré en collision avant d'avoir atteint un réel régime de diffusion $\left({ }^{1}\right)$. Aussi nous avons choisi de remplacer le critère (4) par la comparaison des temps moyens entre collisions déterminés expérimentalement et calculés à partir de la valeur de cisaillement. Sur la même série de clichés où nous avions mesuré les cisaillements, nous avons trouvé que le temps entre collisions pour un amas donné est d'environ $2 \times 10^{3} \mathrm{~s}$. En supposant la distance entre amas de l'ordre de grandeur de la dimension des amas (ce qui correspond au cas de notre expérience, nous sommes au voisinage de la concentration $\phi_{c}$ ) le temps entre collisions induites par le cisaillement est simplement l'inverse du cisaillement, soit $10^{3} \mathrm{~s}$. Il en résulte que l'équivalent du mouvement brownien dans notre expérience est négligeable et donc que le processus d'agrégation étudié est de type orthocinétique avec un cisaillement non contrôlé.

\section{Propriétés d'homothétie interne.}

Un des aspects frappant de l'observation des amas formés dans notre simulation au cours de l'agrégation (cf. Fig. 1) est l'identité de leurs structures géométriques indépendamment de leurs dimensions c'est-à-dire leur aspect fractal [8]. Afin de rendre ceci plus quantitatif, nous avons étudié la variation de l'anisotropie et du diamètre moyen des amas en fonction du nombre de billes constituant l'amas.

Par convention nous avons caractérisé un amas par deux dimensions : $D$ la plus grande dimension et $d$ la dimension mesurée perpendiculairement à la direction de $D$. Le coefficient d'anisotropie $g$ est défini égal au rapport $D / d$ et nous avons représenté sur la figure 3 sa variation avec le nombre des billes constituant l'amas (en échelle semi-log). Une importante dispersion des points est observée autour de la valeur moyenne qui vaut $\bar{g}=1,7$. La valeur de $g$ est typiquement comprise entre 1 et 3,3 avec un écart quadratique moyen de 0,48 (la statistique a été faite sur 243 amas).

Il est important de souligner qu'aucune variation notable de $g$ n'apparaît avec le nombre de billes dans l'amas ( $n$ varie de 1 à 3400 ). Ceci constitue une preuve très claire de l'homothétie interne des objets formés. Par ailleurs nous avons étudié la variation du diamètre des amaś, défini comme la moyenne arithmétique des dimensions $D$ et $d$, avec le nombre de billes dans l'amas. Les points obtenus sont représentés sur la figure 4 en échelle log-log. Nous observons une loi

( $\left.{ }^{1}\right)$ On pourrait penser qu'il suffit pour étudier le mouvement brownien de diminuer la concentration des objets. En fait la situation n'est pas aussi simple car le mouvement des objets est créé par les mouvements de la surface dus aux parasites extérieurs; or ces mouvements de surface dépendent fortement de la façon dont la surface est couverte par les billes. Une répartition du type de celle de la photo 1 donne des mouvements très différents du cas où l'on aurait une seule bille ou un petit amas. 

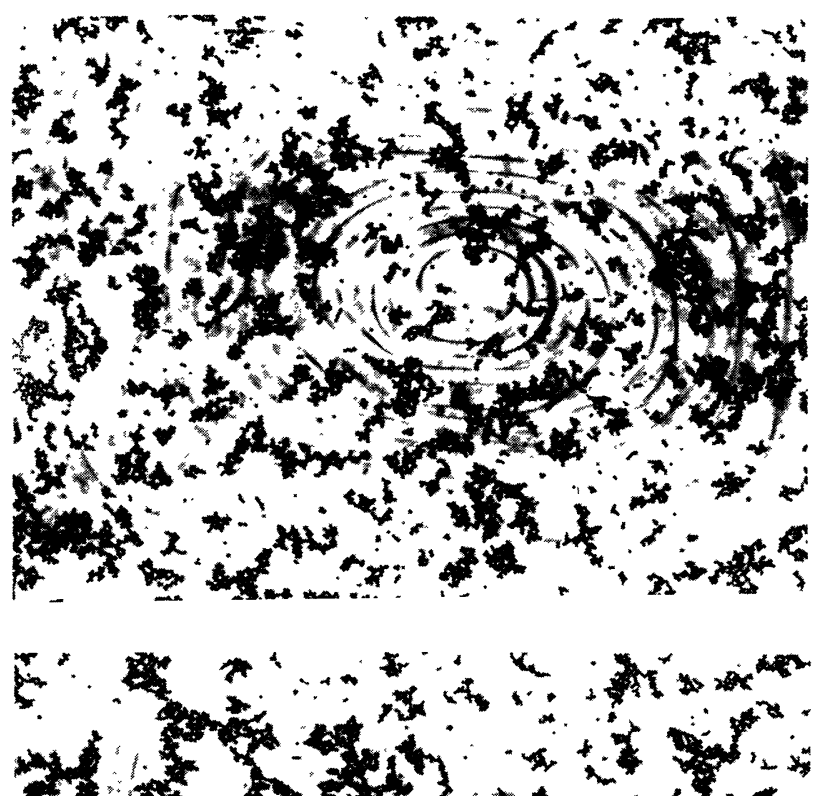
(5)

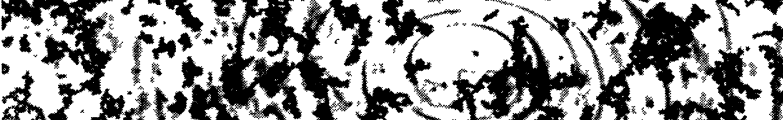
3 for

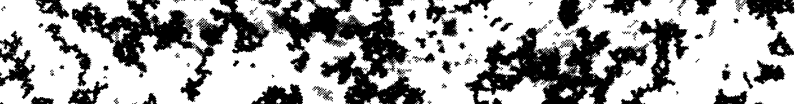

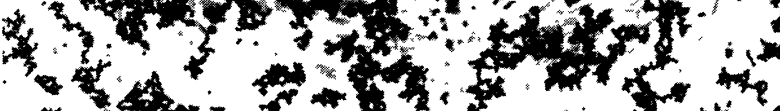
-

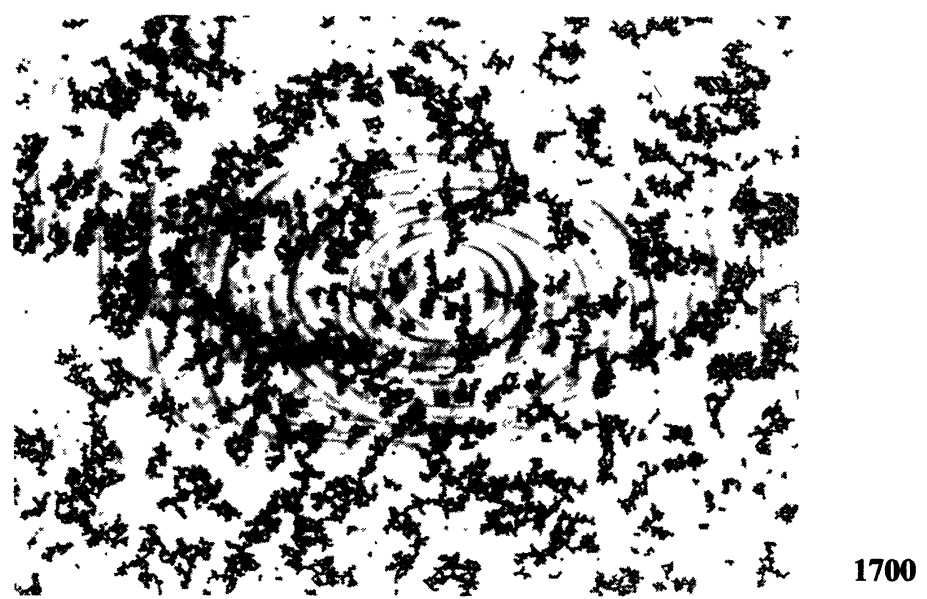

Fig. 2. - Clichés pris au cours de la même expérience à différents temps : 500,1 000, $1700 \mathrm{~s}$. On observe la formation progressive d'amas de plus en plus étendus.

[Pictures taken during the same experiment at different times : 500,1 000, $1700 \mathrm{~s}$. A progressive formation of clusters of increasing size is observed.] 
ANISOTROPIE $\mathrm{G}=\mathrm{D} / \mathrm{d}$

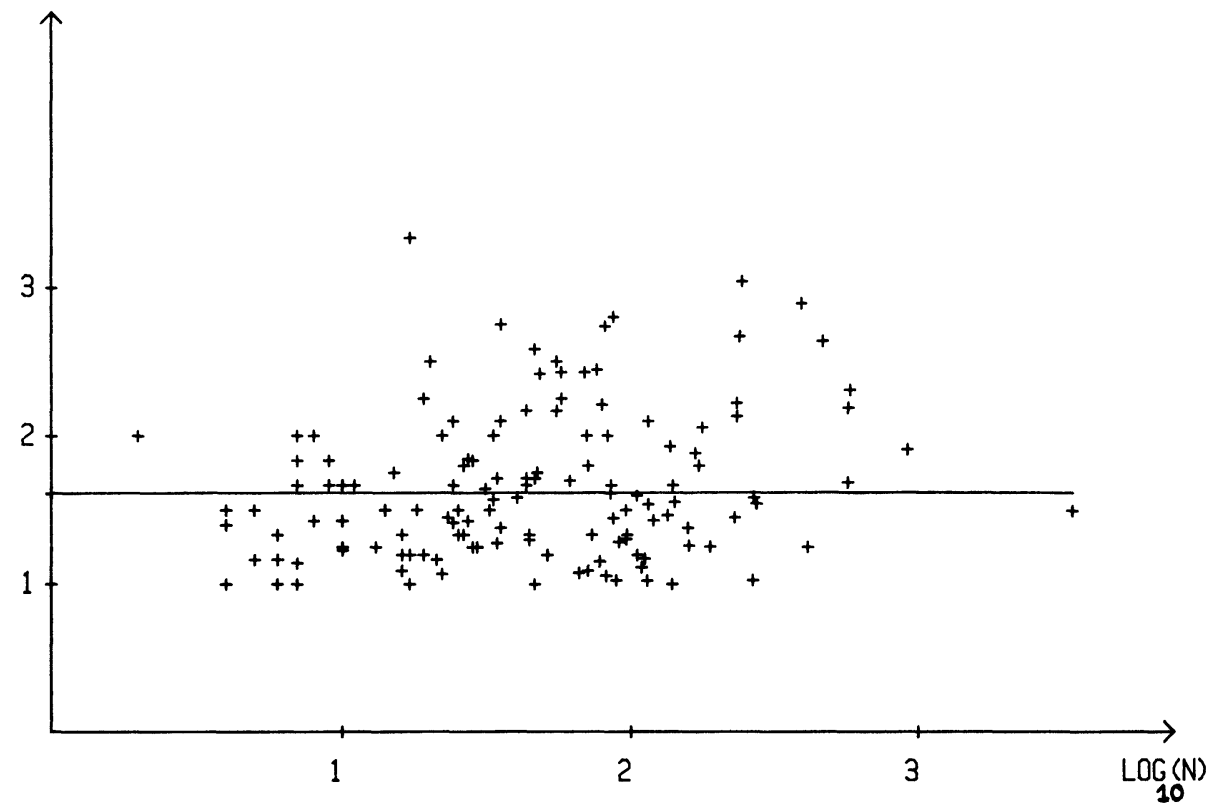

Fig. 3. - Variation en échelle semi-logarithmique de l'anisotropie des amas en fonction du nombre de billes les constituant.

[Semi-log plot of the variation of the cluster anisotropy versus the number of their constitutive balls.]

de puissance bien vérifiée sur trois décades avec un exposant $0,61 \pm 0,05$ ce qui confirme l'homothétie interne des amas montrée par l'étude du coefficient d'anisotropie $\left({ }^{2}\right)$.

Des propriétés analogues d'homothétie interne ont été trouvées sur les amas formés en percolation au voisinage de la probabilité critique d'occupation des sites. Cependant compte tenu des conditions de notre expérience (agrégation orthocinétique avec un cisaillement non contrôlé) l'exposant trouvé ne se compare pas bien avec les valeurs de la littérature. Rappelons simplement pour mémoire que les simulations de percolation [10] au voisinage du seuil de percolation prévoit un exposant de 0,52 et que les statistiques d'animaux [11] conduisent à un exposant valant : 0,641.

Citons également la simulation numérique de l'agrégation limitée par diffusion faite par Witten et Sander [12] (un seul amas est construit sur un réseau triangulaire) qui donne une valeur d'exposant de $0,59 \pm 0,02$.

\section{Conclusion.}

La simulation cinétique de l'agrégation que nous avons faite à deux dimensions montre l'existence, d'une part, d'une fraction -surfacique critique $\phi_{c}$ d'apparition d'un amas de la dimension du récipient, d'autre part de propriétés géométriques d'homothétie interne des amas formés pour une fraction surfacique voisine de $\phi_{\mathrm{c}}$.

$\left({ }^{2}\right)$ La définition du diamètre d'un amas comme étant $(D+d) / 2$ peut sembler simpliste par rapport aux dimensions qui interviennent usuellement comme le rayon de giration $\left(\sum_{i=1}^{n} r_{i_{j}}^{2}\right)^{1 / 2}$ ou le rayon hydrodynamique $\left(\sum_{i=1}^{n} \frac{1}{r_{i_{j}}}\right)^{-1}$. Cependant dans la mesure où les objets présentent des propriétés d'anisotropie indépendante de leur dimension on peut supposer que ces quantités sont proportionnelles à un facteur numérique près. Nous n'avons d'ailleurs observé aucune variation significative de la valeur de l'exposant lorsque nous avons remplacé la moyenne arithmétique par les moyennes d'ordre 2 et d'ordre 3 dans le calcul du diamètre. Remarquons que le même type de résultat a été observé sur les amas générés en percolation [9]. 


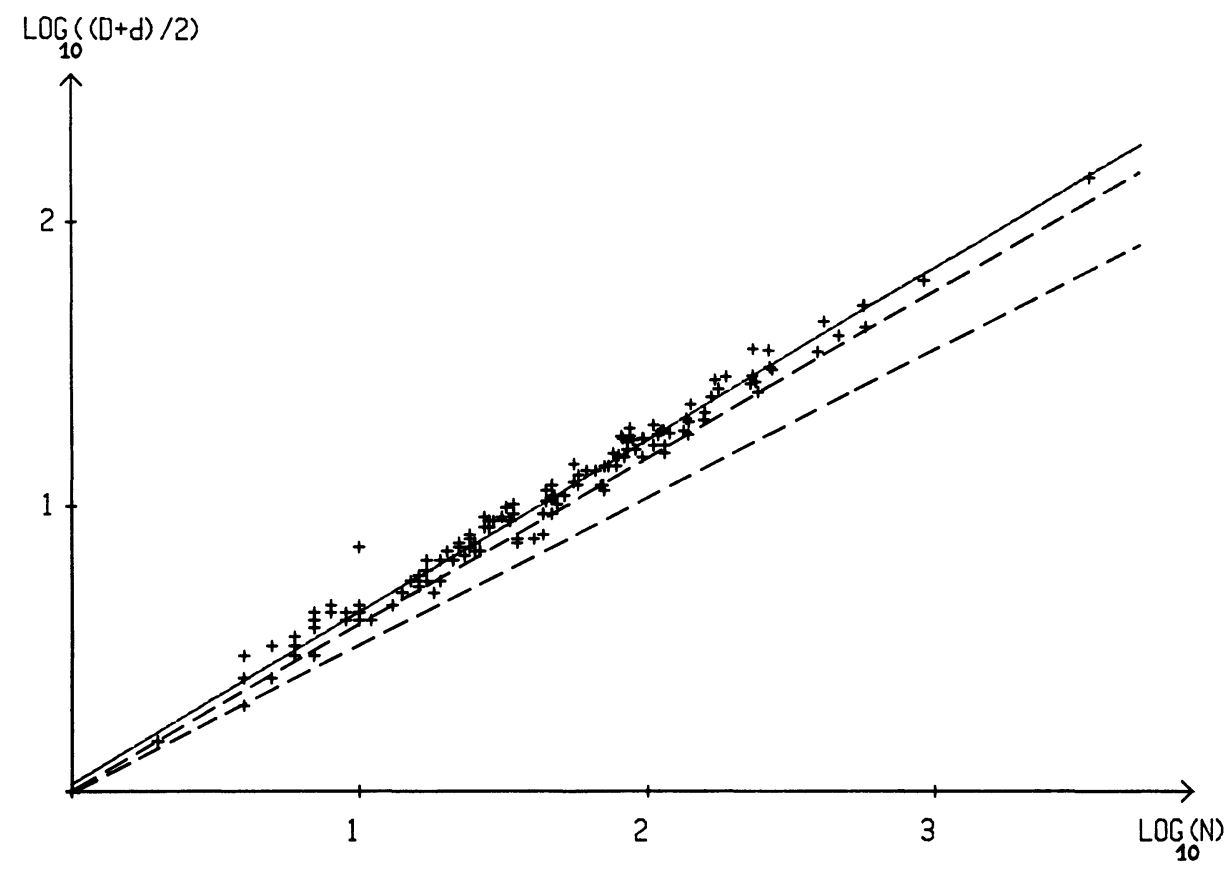

Fig. 4. - Variation en échelle logarithmique du diamètre moyen des amas en fonction du nombre de billes les constituant. La droite en trait plein correspond à un ajustement de courbe fait sur 149 amas et correspond à un exposant de 0,61 . Remarquons que, même pour les très faibles valeurs de $n(1,2)$, l'accord entre les valeurs expérimentales et la loi de puissance est correct. Les droites représentées en pointillé correspondent à des valeurs d'exposant de 0,59 et 0,52 .

[Log plot of the variation of the mean diameter of the cluster versus the number of constitutive balls. The straight line is the result of a fit made on 149 clusters and corresponds to an exponent equal to 0.61 . Notice that, even for the very low value of $n(1,2 \ldots)$, the agreement between the experimental values and the power law is good. The dashed straight lines correspond to exponents equal to 0.59 and 0.52 .]

On peut se demander au terme de cette étude comment arrive-t-on à former avec des mouvements aussi peu contrôlés des amas possédant des propriétés aussi remarquables d'homothétie interne. Il serait intéressant de savoir si cela est dû à un artefact de notre simulation ou si les systèmes réels soumis au même type de conditions comme la formation de flocs dans un agitateur usuel possèdent les mêmes caractéristiques d'homothétie interne.

Dans les équations de type Smoluchowski écrites au début de cette lettre, les coefficients cinétiques ne sont caractérisés que par les nombres $i$ et $j$ sans référence à un facteur de forme. Cette hypothèse est justifiée dans le cas de notre expérience puisque les amas formés possèdent des propriétés fractales. Cependant ces équations ne prennent pas en compte les corrélations spatiales et un modèle théorique incluant l'ensemble de ces propriétés permettrait certainement de bien meilleures prévisions en particulier dans le cas des systèmes concentrés.

\section{Remerciements.}

Ce travail fait suite aux expériences préliminaires effectuées au laboratoire par V. Paul Boncour et $\mathrm{S}$. Alperine que nous remercions vivement. Nous tenons à remercier également $\mathrm{E}$. Guyon pour l'intérêt constant qu'il a porté à ce travail et pour les fructueuses discussions que nous avons eues avec lui, ainsi que P. G. de Gennes, T. Lubensky et T. Witten. 


\section{Bibliographie}

[1] Smoluchowski, M. V., Z. Phys. Chem. 92 (1917) 129 ; Phys. Z. 17 (1916) 585.

[2] Drake, R. L., J. Atmos. Sci. 29 (1972) 537.

[3] Schulthess, G. K., BenedeK, G. B. and Deblois, R. W., Macromolécules 13 (1980) 939.

Ernst, M. H., Hendriks, E. M. and ZifF, R. M., J. Phys. Lett. 15 (1982) L743.

[4] ARp, P. A. and Mason, S. G., Can. J. Chem. 54 (1976) 3769.

[5] Voir par exemple Ives, K. J., The Scientific basis of flocculation, édité par Ives K. J. (Nato advanced study institutes series).

[6] Bruhat, G., Mécanique, Chap. XX (Masson et Cie éditeurs, Paris), p. 460.

[7] Voir par exemple Stauffer, D., Phys. Rep. 54 (1979) 1.

[8] Mandelbrot, B., Fractals, Form, Chance and Dimension (Freeman, San Francisco) 1977.

[9] Stauffer, D., Coniglio, A. and Adam, M., Adv. Polym. Sci. 44 (1982) 103.

[10] FAMily, F. and ReYNolds, P. J., Z. Phys. B. 45 (1981) 123.

[11] Derrida, B. and Vannimenus, J., J. Physique Lett. 41 (1980) L-473.

[12] Witten, T. A. and SANDer, L. M., Phys. Rev. Lett. 47 (1982) 1400. 